Zum Tag der Kranken, 7. März 2004

Kranksein heisst auch gepflegt werden. Am Tag der Kranken soll hier auch der Angehörigen gedacht werden, welche ihre Kranken zu Hause pflegen.

\title{
Angehörige zu Hause pflegen: Anatomie einer Arbeit
}

\section{A. Kesselring}

In der Schweiz werden schätzungsweise 220000-250000 Menschen durch Angehörige zu Hause gepflegt. In einem stark auf das (kranke) Individuum ausgerichteten «Gesundheits»-System wird pflegenden Angehörigen kaum öffentliche, aber auch wenig persönliche Aufmerksamkeit und Anerkennung zuteil. Für die Beschreibung der Arbeit pflegender Angehöriger wird internationale, interdisziplinäre Literatur beigezogen. Der Artikel schliesst mit der Forderung nach Umdenken und bewussterem Erfassen der Situation pflegender Angehöriger.

Korrespondenz:

Annemarie Kesselring PhD, RN Universität Basel

Institut für Pflegewissenschaft Bernoullistrasse 28

$\mathrm{CH}-4056$ Basel

E-Mail:

Annemarie.Kesselring@unibas.ch Ambiguität, z.B. bei Persönlichkeitsveränderungen beim Patienten [3], wenn die Beziehung zum Patienten oder zu anderen Familienmitgliedern schwierig ist, wenn sich der Zustand des Patienten verschlechtert oder grosse Opfer an Freizeit und der eigenen Lebensqualität abverlangt werden [4]. Pflegende Frauen fühlen sich tendenziell mehr belastet als pflegende Männer. Als befrie- digend und sinnstiftend wird Pflege erfahren, die auf Liebe zum Patienten gründet und in der es möglich ist, Erfahrungen zu teilen, Dinge gemeinsam zu tun oder sie so zu bereichern, dass sie auch Freude macht $[5,6]$.

\section{Pflegearbeit}

Die Pflegearbeit hängt von den Aufgaben ab, welche durch die Krankheit oder Behinderung des gepflegten Menschen anfallen. Die Arbeit ist komplex. Es können Fertigkeiten wie Handanlegen, Überwachen, Antizipieren, Vorbeugen, Schützen gefragt sein [7]. Dennoch beinhaltet sie - wenn sie eingeübt ist - viel Routine und ist oft nur soweit plan- und antizipierbar, als sich der Zustand und das Verhalten des Patienten voraussagen lassen. Die Arbeit verlangt von der Pflegenden Lern- und Anpassungsfähigkeit, Kreativität und Einfühlungsvermögen, Umgang mit negativen Gefühlen (z.B. Ekel, Widerwillen, Schuld) sowie viel Toleranz. Oft wird durch Versuch und Irrtum gelernt; es gilt, sich an Veränderungen des Gesundheitszustandes des Patienten anzupassen, Beziehungsarbeit zu leisten und schwierige Symptome bzw. Verhaltensweisen des Patienten zu tolerieren. Besonders schwierig zu tolerieren sind Stuhl- und Urinprobleme, stete Sturzgefahr, Schlafstörungen und Kommunikationsschwierigkeiten. Hohe Anforderungen an die Toleranz der Pflegenden stellen auch gefährliche, apathische, depressive, unkooperative, ruhelose Verhaltensweisen des Patienten und Herumirren $[8,9]$.

\section{Arbeitsbedingungen}

Vergleiche mit Arbeitsbedingungen in der Erwerbsarbeit mögen hinken. Sie helfen jedoch, wesentliche Konturen der Pflegearbeit zu Hause klarer zu umreissen. 


\section{Zeiteinsatz}

Häufig ist der Zeiteinsatz unbegrenzt, umfasst Tag- und Nachtarbeit, mit minimaler bzw. keiner Frei- und Ferienzeit.

\section{Soloarbeit}

Teamarbeit ist selten. In der Regel pflegt eine Person allein und hat wenig oder keinen Austausch mit andern Gleichbeschäftigten.

\section{Stabiles Arbeitsarrangement}

Langzeitpflegen, die sich über Jahre erstrecken, sind häufig, vor allem dann, wenn Pflegende und Patient die erste, schwierige Zeit des Lernens und Gewöhnens überstanden haben.

\section{Alter}

Es gibt keine Altersbeschränkungen für pflegende Angehörige. In Afrika pflegen Kinder ihre AIDS-kranken Eltern [10] und hierzulande pflegen auch Personen, die über 90 Jahre alt sind. Viele Pflegende gelten offiziell als im «Ruhestand» [11].

\section{Vergütung}

Finanzielle oder materielle Vergütungen für die Pflegende sind die Ausnahme und wo bezahlt oder materiell vergütet wird, ist es vergleichsweise wenig. Oft reduzieren jüngere Pflegende ihre Erwerbsarbeit oder geben sie ganz auf, um pflegen zu können. Dadurch erleiden sie Einbussen in ihrer Altersvorsorge.

\section{Arbeitsbefriedigung}

Wenn Besserung oder gar Genesung als Resultat einer Pflege angestrebt wird, bringt die Arbeit selten befriedigende Resultate. Häufig nehmen Pflegeaufwand und persönliche Anstrengung in dem Masse zu, wie's dem Patienten schlechter geht. Die gängige Erwartung, dass mit Mehraufwand bessere Resultate erzielt werden, wird in ihr Gegenteil verkehrt.

\section{Karriere}

Tendenziell vereinnahmt die Karriere - je länger sie dauert - mehr und mehr Kraft, Zeit und Lebensbereiche der Pflegenden; Aufstiegsmöglichkeiten gibt es jedoch keine.

\section{Soziales Prestige}

Die Arbeit geniesst kein hohes gesellschaftliches Prestige. In den Familien und auch bei Ärzten und Pflegenden gilt sie meist als selbstverständlich und erntet kaum Anerkennung.

\section{Arbeiterinnen}

Zu 70-80\% sind es Frauen, die Angehörige pflegen. Frauen pflegen ihre Eltern, Partner, Kinder und Freunde, während Männer vornehmlich ihre Partnerin pflegen. Männer pflegen eher «freiwillig», wenn die Beziehung zur gepflegten Person gut ist; sie sind auch eher bereit, auf eigene Bedürfnisse zu achten und Hilfe anzunehmen als Frauen. Von Frauen wird vielfach erwartet, dass sie pflegen, auch wenn die Beziehung zur gepflegten Person nicht so gut ist. Vielen Frauen fällt es schwer, sich gegenüber den Erwartungen anderer abzugrenzen, eigene Bedürfnisse wahrzunehmen sowie Hilfe und Entlastungsangebote anzunehmen. Es mag sein, dass dies Gründe sind, warum sich Frauen durch die Pflege tendenziell mehr belastet fühlen als Männer.

\section{Gesundheit}

Vorsicht ist geboten beim Herleiten kausaler Bezüge zwischen zwei so komplexen Phänomenen wie Gesundheit und Pflege. Dennoch: Viele pflegende Angehörige leiden signifikant häufiger an Depressionen und Schlafstörungen sowie unter Kopf-, Rücken- und Gelenkschmerzen als Gleichaltrige, die nicht pflegen $[12,13]$.

\section{Was können Ärzte und Pflegende tun?}

Die Hilfe, Unterstützung und Betreuung pflegender Angehöriger gehört in den Aufgabenbereich von Ärzten und Pflegenden. Vielfach wird die geleistete Pflegearbeit kaum bemerkt oder nur, wenn etwas nicht klappt: dann wird sie bemängelt. Es braucht professionelles Umdenken und - für die Betroffenen - annehmbare (private) und professionelle Angebote.

\section{Umdenken}

Auf seiten der Ärzte und der Pflegenden ist doppeltes Umdenken gefordert:

1. Kranksein ist keine individuelle Angelegenheit; es betrifft immer auch jene, die einem Menschen nahestehen. Wo jemand pflegebedürftig ist, pflegt jemand. Pflegende und Gepflegter sind ein System, in dem die subjektiv erfahrene Situation und der Gesundheitszustand der pflegenden Angehörigen von zentraler Bedeutung für die Kontinuität der Pflege zu Hause sind. 
2. Umdenken im Bereich geschlechtlicher Gleichberechtigung tut auch not: Männer sind zur Pflege so fähig - und so berechtigt wie Frauen! Entsprechend ist die Pflege durch eine Frau genausowenig selbstverständlich, aber genauso grossartig, wie wenn ein Mann pflegt.

\section{Professionelle Angebote}

Vorerst und vor allem gilt es, pflegende Angehörige und die von ihnen geleistete, grosse Arbeit anzuerkennen. Sie brauchen Gelegenheiten, sich über ihr Befinden und die Pflege auszusprechen, sich informieren und beraten zu lassen.

Niederschwellige, für die Betroffenen annehmbare, situationsadäquate Hilfs-, Entlastungs- und Unterstützungsangebote sind notwendig [14]. Dabei gilt das besondere Augenmerk dem Gesundheitszustand (z.B. Depression, Schlafmangel) sowie der Entlastung (z.B. Ablösung, Nachtruhe, Ferien, Bereicherung der Pflege, Alternativen im Umgang mit schwierigen Symptomen und Verhaltensweisen des Patienten) der pflegenden Angehörigen.

Ärzte und Pflegende sind die primären, professionellen Kontaktpersonen für pflegende Angehörige. Oft ist in einer Begegnung wenig mehr möglich, als zuzuhören und anzuerkennen, was körperlich und seelisch geleistet wird. Das ist bereits viel mehr, als für selbstverständlich genommen zu werden.

\section{Literatur}

1 Pearlin LI, Mullan JT, Semple SJ, Skaff MM. Care giving and the stress process: an overview of concepts and their measures. Gerontologist 1990;30(5):583-94.

2 Hirschfeld MJ. Home care versus institutionalization: family care giving and senile brain disease. Int J Nurs Stud 1983;20(1):23-32.

3 Boss P, Caron W, Horbal J, Mortimer J. Predictors of depression in caregivers of dementia patients: boundary ambiguity and mastery. Fam Process 1990;29(3):245-54.
4 Jani-Le Bris H. Family care of dependent older people in the European Community. European Foundation for the Improvement of Living and Working Conditions. Dublin: Loughlinstown House, Shankil, Co.; 1993.

5 Archbold PG, Stewart BJ, Merwyn R, Greenlick MR, Harvarth T. Mutuality and preparedness as predictors of caregiver role strain. Res Nurs Health 1990;13(6):375-84.

6 Archbold PG, Stewart BJ, Miller LL, Harvarth TA, Greenlick MR, Van Buren L, et al. The PREP system of nursing interventions: a pilot test with families caring for older members. Res Nurs Health 1995;18(1):3-16.

7 Schumacher KK, Stewart BJ, Archbold PG. Conceptualization and measurement of doing family care giving well. Image J Nurs Sch 1998;30(1): 63-9.

8 Krulik T, Hirschfeld MJ. The continuation of homecare to severely impaired children and aged in Israel: family attitudes. Home Health Care Quarterly 1985:5(3,4):283-313.

9 Kesselring A, Krulik T, Bichsel M, Minder C, Beck JC, Stuck AE. Emotional and physical demands on caregivers in home care to the elderly in Switzerland and their relationship to nursing home admission. Eur J Public Health 2001;11(3):267-73.

10 World Health Organization. Community Home Based Care: Action Research in Kenya. Geneva: WHO; 2001.

11 Doran T, Drever F, Whitehead M. Health of young and elderly informal carers: analysis of UK census data. Br Med J 2003;327:1388.

12 Schulz R, O'Brien AT, Bookwala J, Fleissner K. Psychiatric and physical morbidity effects of dementia caregiving: prevalence, correlates, and causes. Gerontologist 1995;35(6):771-91.

13 Schulz R, Mendelsohn AB, Haley WE, Mahoney D, Allen RS, Zhang S, et al. End-of-life care and the effects of bereavement on family caregivers of persons with dementia. N Engl J Med 2003;349(20):1936-42.

14 Schweizerisches Rotes Kreuz. Pflegend begleiten: Ein Alltagsratgeber für Angehörige und Freunde älterer Menschen. Bern: Schweizerisches Rotes Kreuz, Nationales Sekretariat der Kantonalverbände; 2001. 